\title{
Afectación hepática y cutánea gomatosas simultáneamente en la presentación de sífilis tardía en paciente no coinfectado por el VIH
}

\author{
Rubén Fortea-Carrasco', Diana Lucía Tarruella-Hernández², Ana Isabel Renau-Escrig ${ }^{3}$, Miguel Salavert-Lletí ${ }^{4}$ \\ ${ }^{1}$ Servicio de Medicina Intensiva, Hospital Universitari i Politècnic La Fe, Valencia, España \\ ¿2Servicio de Neurología, Hospital Universitari i Politècnic La Fe, Valencia, España \\ ${ }^{3}$ Servicio de Medicina Interna, Hospital Universitari i Politècnic La Fe, Valencia, España \\ ${ }^{4}$ Unidad de Enfermedades Infecciosas, Hospital Universitari i Politècnic La Fe, Valencia, España
}

Recibido: 04/02/2021

Aceptado: 28/03/2021

En línea: 30/04/2021

Citar como: Fortea-Carrasco R, Tarruella-Hernández DL, Renau-Escrig Al, Salavert-Lletí M. Afectación hepática y cutánea gomatosas simultáneamente en la presentación de síflis tardía en paciente no coinfectado por el VIH. Rev Esp Casos Clin Med Intern (RECCMI). 2021 (abril); 6(1): 43-46. doi: 10.32818/reccmi.a6n1a13.

Cite this as: Fortea-Carrasco R, Tarruella-Hernández DL, Renau-Escrig Al, Salavert-Lletí M. Gummatous liver and skin involvement simultaneously as first manifestation of late syphilis in a patient not infected with HIV. Rev Esp Casos Clin Med Intern (RECCMI). 2021 (April); 6(1): 43-46. doi: 10.32818/reccmi.a6n1a13.

Autor para correspondencia: Rubén Fortea-Carrasco. rubenforteacarrasco@hotmail.com

Palabras clave
$\triangleright$ Sífilis terciaria
$\triangleright$ Microabscesos hepáticos
$\triangleright$ Goma sifilítica
$\triangleright$ Treponema pallidum

\section{Keywords}

$\triangleright$ Tertiary syphilis

$\triangleright$ Liver microabscesses

$\triangleright$ Syphilitic gum

$\triangleright$ Treponema pallidum

\begin{abstract}
Resumen
Lejos de estar erradicada, la infección sifilítica es hoy en día un problema creciente, que afecta principalmente a la población joven. De los pacientes con infección sifilítica sin tratamiento, aproximadamente entre el 25-40\% desarrollan síflis tardía. Es particularmente extraña la afectación sifilítica del hígado, que se aprecia especialmente en pacientes con coinfección por el VIH.

Se presenta el caso de un paciente de 33 años con sífilis terciaria con afectación cutánea en forma de goma y hepática en forma de microabscesos, que se resolvió tras completar tratamiento endovenoso.
\end{abstract}

Abstract
Far from being eradicated, syphilitic infection is now a growing problem, mainly affecting the young popu-
lation. Approximately 25-40\% of patients with syphilitic infection without treatment, develop late syphilis.
Syphilitic involvement of the liver is particularly rare, especially seen in patients with HIV coinfection.
We present the case of a 33-year-old patient with tertiary syphilis with skin involvement in the form of gum
and liver involvement in the form of microabscesses, which resolved after completing intravenous treatment.

\section{Puntos destacados}

$\triangleright \quad$ La incidencia de la infección sifilítica está creciendo en los últimos años, observándose incluso formas tardías de la misma.

$\triangleright \quad$ La afectación hepática de la sífilis es poco frecuente y menos aún en pacientes sin coinfección por el VIH.

\section{Introducción}

La sífilis constituye una enfermedad infecciosa de transmisión principalmente sexual, producida por la espiroqueta Treponema pallidum. Puede manifestarse clínicamente como sífilis primaria, secundaria, latente o terciaria (tardía).

Según la Organización Mundial de la Salud (OMS), en 2016 hubo 19,9 millones de casos de sífilis entre los 15 y los 49 años, y 6,3 millones de nuevos casos ${ }^{2}$. Actualmente, se registra un incremento de esta y otras infecciones de transmisión sexual en nuestro medio. En 2017 se notificaron 4.941 nuevos casos de sífilis, lo que supuso una tasa de 10,61 por 100.000 habitantes, mostrando una tendencia creciente desde el año 2000. El 88,9\% (3.863) fueron varones y la razón hombre/mujer fue de 8,0, con una mediana de edad de 37 años $^{3}$. Entre el 25 y el $40 \%$ de los pacientes sin tratamiento desarrollan síflis tardía 4 .

La sífilis puede afectar al hígado, tanto en forma de hepatitis sifilítica en la sífilis secundaria, como en forma de goma, durante la sífilis terciaria². La afectación hepática gomatosa es rara en la sífilis terciaria, más frecuente en pacientes con coinfección por el VIH, y se describe usualmente en las necropsias ${ }^{4}$.

\section{Caso clínico}

Antecedentes y enfermedad actual

Se presenta el caso de un varón de 33 años, fumador de 9 paquetes/año, con prácticas sexuales de riesgo (receptor de penetración anal sin protec- 
Fortea-Carrasco R, Tarruella-Hernández DL, Renau-Escrig Al, Salavert-Lletí M.

Afectación hepática y cutánea gomatosas simultáneamente en la presentación de sífilis tardía en paciente no coinfectado por el VIH

ción); sífilis primaria y secundaria ya tratadas y con serología negativa para la infección por el VIH. Su último control de RPR era de 1/2, un año antes de la fecha del ingreso. Trabajaba de cajero en un supermercado y también padeció gonorrea en el pasado. El paciente ingresa en la Unidad de Enfermedades Infecciosas por sospecha de sífilis terciaria como goma cutánea y microabscesos hepáticos.

Tres semanas antes del ingreso presentó un cuadro de fiebre de hasta $39^{\circ} \mathrm{C}$ con escalofríos, de predominio nocturno, que remitía con ibuprofeno. Asociaba astenia e hiporexia sin pérdida ponderal, dolor abdominal de localización en hipocondrio derecho y expectoración de escasa cuantía. Negaba nuevas relaciones sexuales de riesgo.

\section{Exploración física y pruebas complementarias}

Fue valorado en consultas externas, observándose una lesión nodular en nuca de unos $2 \mathrm{~cm}$ como único hallazgo exploratorio. Se solicitó una TC toraco-abdominopélvica (TAP) (Figura 1) donde se apreciaban lesiones hepáticas centimétricas sugestivas de granulomas caseosos o microabscesos por probable síflis terciaria gomosa.

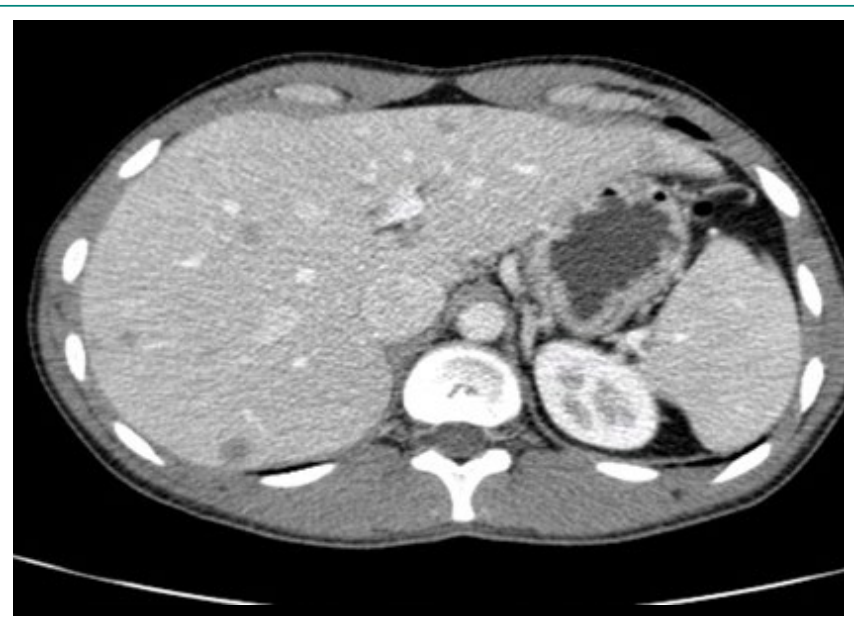

Figura 1. TC toraco-abdominopélvica en el momento del diagnóstico: varias lesiones hipodensas de distintos tamaños en el parénquima hepático que corresponden a pequeños abscesos.

Así mismo se solicitaron nuevas serologías sifilíticas, siendo el RPR 1/32 y analítica ordinaria en la que únicamente destacaba un patrón de colestasis disociada con discreto aumento de GGT y fosfatasa alcalina (FA) (GGT $71 \mathrm{U} / \mathrm{L}$, FA $152 \mathrm{U} / \mathrm{L}), \mathrm{LDH}$ de $192 \mathrm{U} / \mathrm{L}$, así como un mínimo incremento de reactantes de fase aguda (PCR de 20,2 mg/L).

Con el resultado de la TC y ante la persistencia de fiebre, se ingresó al paciente para ampliar el estudio e iniciar tratamiento.

A su ingreso se realizaron biopsia hepática guiada por ecografía (Figura 2), biopsia de la lesión cutánea, junto con cultivos de esputo, y se inició tratamiento con penicilina G sódica a dosis de 20 millones de unidades (MU) intravenosa (i.v.) en infusión continua.

No fue posible realizar estudio complementario de LCR mediante punción lumbar tras varios intentos fallidos.

Se descartó infección tuberculosa (prueba de IGRA-Quantiferon TB- negativa) y por el $\mathrm{VIH}$, concomitantes. En las analíticas realizadas persistía una leve elevación de GGT, FA y reactantes de fase aguda, que se normalizaron tras completar el tratamiento antibiótico i.v. durante 10 días, encontrando el resto de parámetros dentro de la normalidad.

En las muestras microbiológicas solo se consiguió detectar DNA de T. pallidum en la lesión cutánea, no así en la biopsia ecoguiada, en la que se obtuvo un cilindro de parénquima hepático con leve inflamación portal, sin identificarse microorganismos.

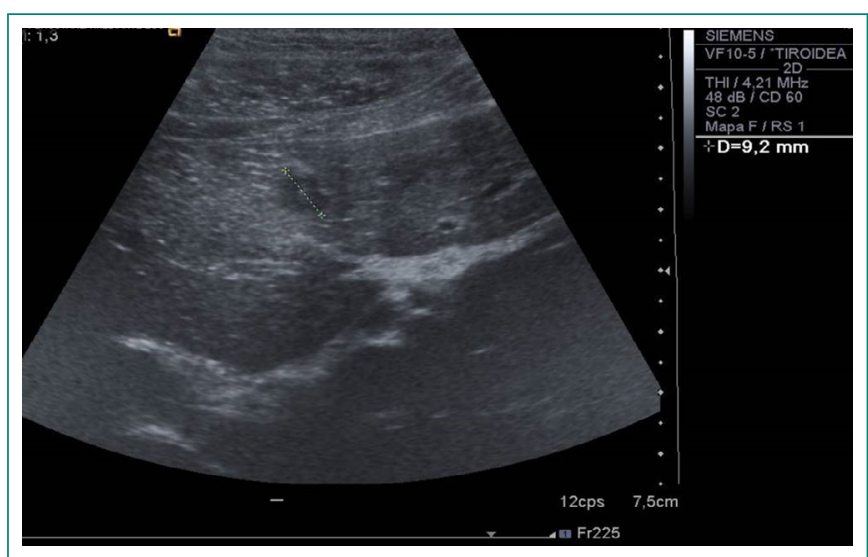

Figura 2. Ecografía hepática realizada para la práctica de biopsia percutánea que mostraba algunas de estas pequeñas lesiones ocupantes de espacio.

\section{Evolución}

La fiebre remitió con el tratamiento antibiótico. Ante la mejoría clínica y analítica, el paciente fue dado de alta tras finalizar la antibioterapia, con recomendaciones para evitar prácticas sexuales de riesgo.

En la cita ambulatoria, un mes después, no había lesiones cutáneas recurrentes o nuevas, y la analítica de control no mostró alteraciones. El estudio de RPR fue de 1/8, apreciándose un descenso precoz tras el tratamiento.

La ecografía (Figura 3) mostró resolución de los abscesos hepáticos descritos en la TC previa al ingreso. En la última consulta, seis meses tras el alta, el paciente estaba asintomático y había negativizado el RPR.

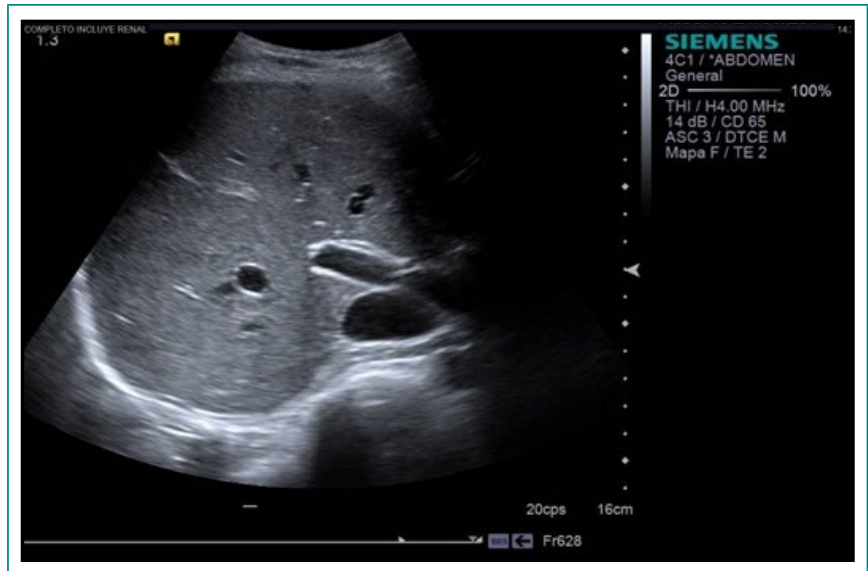

Figura 3. Ecografía hepática de control 1 mes tras alta y finalizado el tratamiento antibiótico, con ausencia de lesiones distinguibles al ultrasonido.

En la Figura 4 se puede apreciar un resumen de la evolución de la enfermedad sifilítica del paciente. 


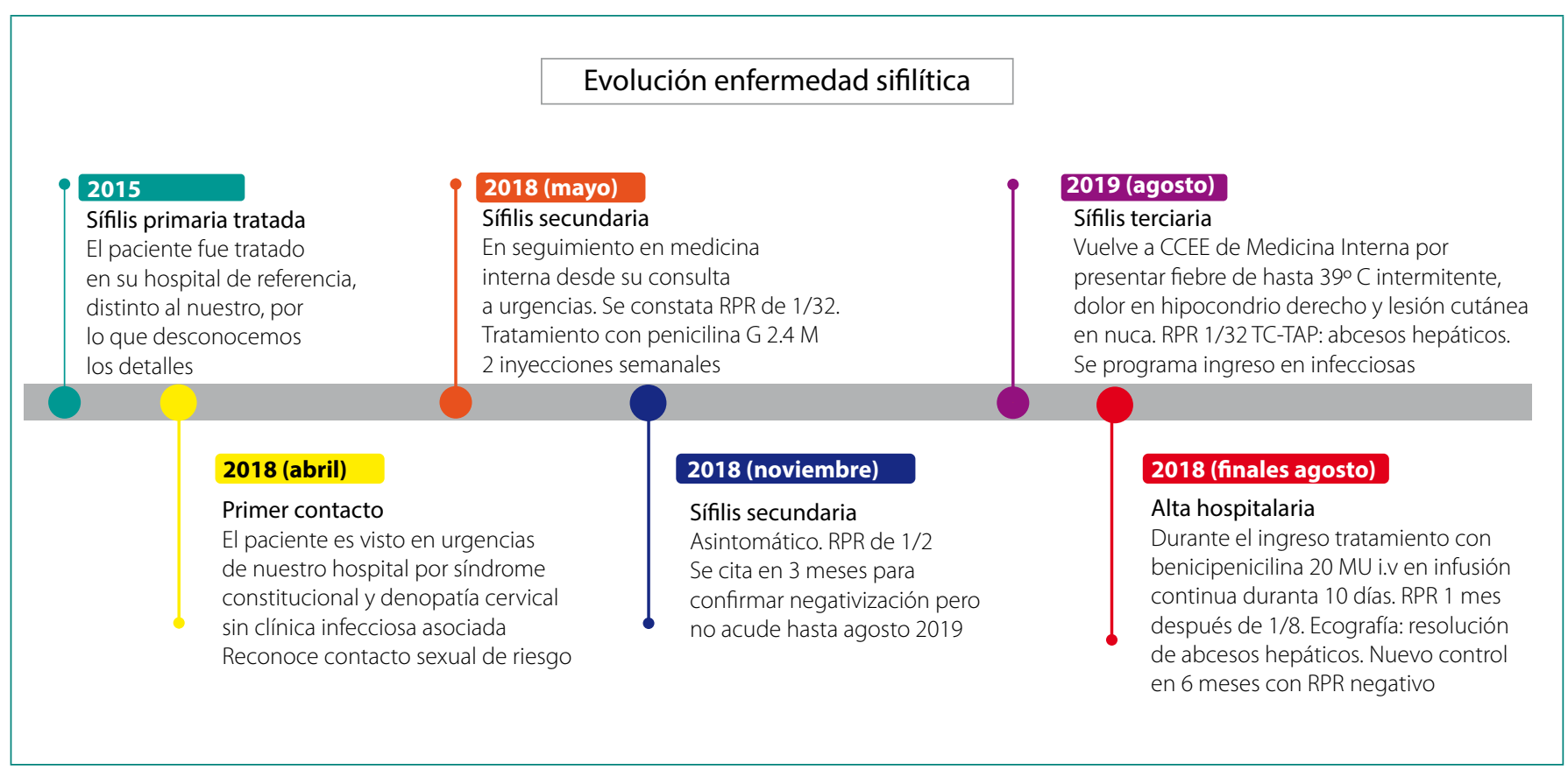

Figura 4. Cronograma de enfermedad sifilítica en el paciente presentado.

\section{Diagnóstico}

Sífilis tardía con afectación hepática y cutánea gomatosas.

\section{Discusión y conclusión}

La afectación gomatosa hepática en la sífilis terciaria es rara, más frecuente en pacientes con coinfección por el VIH, y se describe usualmente en las necropsias. Aproximadamente, se encuentra en el 5\% de las mismas, realizadas en adultos con sífilis en cualquier estadio y en el $16 \%$ en series de pacientes afectos de sífilis terciaria4.

La afectación sifilítica en forma de masas se confunde a menudo con tumores malignos, ya que la imagen es similar y este tipo de afectación es infrecuente. Este hecho comporta que se realice una biopsia, a pesar de no ser necesaria para el diagnóstico, pues los síntomas se resuelven con la administración de antibiótico endovenoso.

Aunque es posible aislar el microorganismo y visualizarlo con técnicas inmunohistoquímicas o tinción de Warthin-Starry, el porcentaje de aislamiento de la espiroqueta en los granulomas varía desde $0-5 \%$ al $50 \%$, siendo mayor en las fases más tempranas de la infección sifilítica ${ }^{4}$. Así pues, para aproximar el diagnóstico, en la literatura se describen factores como la historia de sífilis previa, afectación sifilítica de otros órganos (en especial aortitis y afectación del sistema nervioso central), junto con test serológicos positivos ${ }^{5,6}$. En nuestro caso, de acuerdo con la revisión documental, se realizó el diagnóstico aunando la historia de sífilis previa, la positividad del RPR y el aislamiento del T. pallidum en la biopsia cutánea.

El tratamiento de la sífilis se basa en el estadio clínico en el que se encuentre el paciente. Así, dentro de la sífilis terciaria, varía en función de si existe o no afectación del sistema nervioso central. En caso de haberla, debe ser con penicilina G sódica (3 a 4 MU i.v. cada cuatro horas, o 18 a 24 MU mediante infusión continua) durante 10 a 14 días. En caso de no presentarla, el tratamiento de elección es penicilina G benzatina 2,4 MU intramuscular (i.m.) una vez por semana, durante tres semanas. En alérgicos a penicilina se recomiendan nuevas pruebas de alergia y tratar de desensibilizar al paciente, sobre todo en caso de neurosífilis. Como alternativas podría emplearse doxiciclina o ceftriaxona ${ }^{7}$.

En el caso de nuestro paciente, sin haber podido descartar la afectación neurológica, aunque no existía semiología neuropsiquiátrica, se decidió tratar la infección como si se tratase de una neurosífilis.

La posición de los granulomas, y, en especial, su proximidad o lejanía al hilio hepático, es determinante en el pronóstico de estos pacientes. Más concretamente, cuanto más cercano al hilio, mayor es la posibilidad de generar hipertensión portal y, por tanto, el pronóstico es peor.

Así, son las complicaciones derivadas de la hipertensión portal tales como la hemorragia varicosa, las principales causas de mortalidad, que puede alcanzar hasta un 34\% en algunas series. Sin embargo, con el actual arsenal terapéutico antibiótico, la afectación hepática rara vez concluye con un desenlace fatal ${ }^{6}$, de acuerdo con el caso presentado.

Cabe destacar la rareza de esta forma de presentación de sífilis terciaria con afectación hepática y cutánea simultáneas, en un paciente homosexual, no coinfectado por el VIH y que respondió favorablemente al tratamiento antibiótico. Así mismo, es necesario recalcar la importancia de la prevención, con prácticas sexuales seguras en este grupo de pacientes y en toda la población.

\section{Bibliografía}

1. Clement ME, Okeke NL, Hicks CB. Treatment of syphilis: a systematic review. JAMA. 2014;312(18): 1905-17. doi: 10.1001/jama.2014.13259.

2. Rowley J, Vander Hoorn S, Korenromp E, Low N, Unemo M, Abu-Raddad LJ et al. Chlamydia, gonorrhoea, trichomoniasis and syphilis: global prevalence and incidence estimates, 2016. Bull World Health Organ. 2019;97(8): 548-562P. doi: 10.2471/BLT.18.228486. 
Fortea-Carrasco R, Tarruella-Hernández DL, Renau-Escrig Al, Salavert-Lletí M.

Afectación hepática y cutánea gomatosas simultáneamente en la presentación de sífilis tardía en paciente no coinfectado por el VIH

3. Unidad de vigilancia del VIH y conductas de riesgo. Vigilancia epidemiológica de las infecciones de transmisión sexual, 2017. Madrid: Centro Nacional de Epidemiología, Instituto de Salud Carlos III/Plan Nacional sobre el Sida, Dirección General de Salud Pública, Calidad e Innovación; 2019.

4. Rosahn PD. Autopsy studies in syphilis. 649 Information supplement \#21, J Venereal Disease; U.S. Public Health Service Venereal Disease Division, Washington, DC 1947

5. Hagen CE, Kamionek M, McKinsey DS, Misdraji J. Syphilis presenting as inflammatory tumors of the liver in HIV-positive homosexual men. Am J Surg Pathol. 2014;38(12): 1636-43. doi: 10.1097/PAS.0000000000000264.
6. Maincent G, Labadie H, Fabre M, Novello P, Derghal K, Patriarche C, Licht H. Tertiary hepatic syphilis. A treatable cause of multinodular liver. Dig Dis Sci. . 1997; 42(2): 447-50. doi: 10.1023/a:1018855011180.

7. AIDS Study Group (GESIDA), National Aids Plan; STI Study group of the SEIMC (GEITS); Spanish Group for the Investigation of Sexual Transmission Diseases of the Spanish Academy of Dermatology and Venerology; Spanish Society for Pediatric Infectious (SEIP). Consensus document on the diagnosis and treatment of sexually transmitted diseases in adults, children and adolescents. Enferm Infecc Microbiol Clin. 2018;36(9): 576-585. doi: 10.1016/j.eimc.2017.06.004 